\title{
Organizações Sociais de Saúde: potencialidades e limites na gestão pública*
}

Social Organizations of Health: Potentials and limits of public management

Organizaciones Sociales de Salud: potencialidades y límites en la gestión pública

Clarita Terra Rodrigues ${ }^{1}$, Regina Stella Spagnuolo ${ }^{2}$

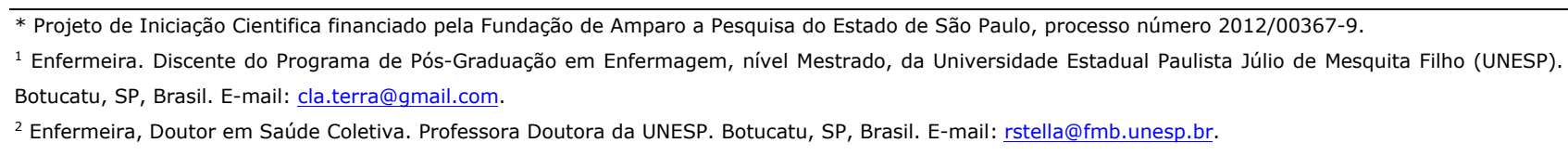

\section{RESUMO}

Este estudo objetivou conhecer o gerenciamento de uma Organização Social de Saúde (OSS) de um município do interior paulista, na perspectiva dos gestores. Trata-se de estudo qualitativo do tipo Estudo de Caso Único, com sete participantes do Conselho de Curadores. Os dados foram coletados por meio de entrevista não diretiva, entre os meses de março e maio de 2012, e analisados segundo análise de conteúdo, resultando em quatro categorias temáticas: 1 . O processo histórico, as transformações sociais e a OSS local; 2 . O contrato de gestão da OSS: agilidade e novos aprendizados; 3. OSS: uma nova possibilidade de gestão; 4. Limitações no gerenciamento e nos mecanismos de controle da OSS local. O estudo deu visibilidade às potencialidades do gerenciamento da OSS, sendo destaques a agilidade dos serviços e a objetividade do contrato de gestão. Apresentaram-se como limitações a escassa participação social e seus mecanismos de controle.

Descritores: Organização Social; Gestão em Saúde; Modelos Organizacionais.

\section{ABSTRACT}

The present study had the aim to become familiar with the Social Organization of Health (OSS, as per its acronym in Portuguese) from a municipality of the state of São Paulo from the managers' viewpoint. It is a qualitative singlesubject study, with seven participants from the Council of Healers. Data were collected through a non-directive interview, resulting in four thematic categories: 1 . The historical process, social transformations and the local OSS. 2. The OSS management contract: agility and new learning. 3. OSS: A new management possibility. 4. Limits of local OSS management and its control mechanisms. The study shed light on the OSS's management potentials, with emphasis on the service's agility and the objectivity of the management contract. As limitations, we point out its control mechanisms and the very low level of social participation.

Descriptors: Social Organization; Health Management; Models, Organizational.

\section{RESUMEN}

Se objetivó conocer el gerenciamiento de una Organización Social de Salud (OSS) de un municipio del interior paulista en la perspectiva de los administradores. Estudio cualitativo, tipo Estudio de Caso Único, con siete participantes del Consejo de Curadores. Datos recolectados mediante entrevista no directiva entre marzo y mayo de 2012, analizados según análisis de contenido, resultando en cuatro categorías temáticas: 1. El proceso histórico, las transformaciones sociales y la OSS local. 2. El contrato de gestión de la OSS: agilidad y nuevos aprendizajes. 3. OSS: una nueva posibilidad de gestión. 4. Limitaciones en el gerenciamiento y mecanismos de control de la OSS local. El estudio hizo visibles las potencialidades del gerenciamiento de la OSS, habiéndose destacado la agilidad de los servicios y la objetividad del contrato de gestión. Se presentaron como limitaciones la escasa participación social y sus mecanismos de control.

Descriptores: Organización Social; Gestión en Salud; Modelos Organizacionales. 


\section{INTRODUÇÃO}

Em busca de maior eficiência e agilidade nos processos de gestão pública, entre os anos de 1995 e 1998, modelos alternativos de administração foram propostos como resultado do Plano Diretor da Reforma do Aparelho do Estado (PDRAE), o qual estabeleceu a existência do Terceiro Setor, como estratégia para a administração pública no Brasil ${ }^{(1)}$.

Neste contexto, surgem como alternativa, as Organizações Sociais (OS) regulamentas pela Lei $n$. 9637/98 definidas como personalidades jurídicas de direito privado, com ações dirigidas ao ensino, pesquisa, desenvolvimento tecnológico, proteção e preservação do meio ambiente, cultura e saúde ${ }^{(2)}$.

As OS podem ser associações sem finalidade lucrativa ou fundações de direito privado, qualificadas pelo poder público para exercer atividade pública descentralizada ${ }^{(2)}$.

Seu principal objetivo é oferecer mais flexibilidade e autonomia ao serviço público, permitindo aumento da eficiência e qualidade do mesmo, sendo fomentadas pelo Estado, o qual tem por obrigação o controle de resultados, via contrato de gestão preestabelecido, que deve conter metas de desempenho e aporte de recursos necessários para execução das atividades pactuadas.

Os regulamentos referentes ao processo de compras, pessoal e administração financeira são próprios e devem constar no contrato de gestão, porém, anualmente, as OS devem prestar contas dos recursos públicos disponibilizados.

Servidores estatutários, podem ser disponibilizados pelo Estado para as OS, bem como a contratação de novos servidores com vínculo ao regime da CLT (Consolidação das Leis do Trabalho) ${ }^{(3)}$.

Este contrato deverá ser avaliado periodicamente pela comissão de avaliação composta por representantes do Legislativo, do Executivo e do Conselho Estadual de Saúde, tendo a responsabilidade de analisar os resultados alcançados ${ }^{(4)}$.

Em meados de 1998, O Estado de São Paulo implantou este modelo de gestão na saúde, com base na Lei Complementar no 846/98, que permite ao Executivo Estadual qualificar entidades sem fins lucrativos e com experiência comprovada no gerenciamento do setor saúde, sendo designadas como Organizações Sociais de Saúde (OSS) ${ }^{(5)}$.

Essas implantações ocorreram por motivos concretos, uma vez que o Estado encontrava-se limitado pela Lei de Responsabilidade Fiscal (Lei Camata), a qual impossibilitava a contratação de novos profissionais de saúde pelo Estado(6).

Ao nível estadual este fato foi visto como uma das experiências mais bem avaliadas nos últimos anos no setor saúde, evidenciado em estudo realizado pelo Banco Mundial no ano de 2006, onde apontou que os hospitais gerenciados pelas OSS são $30 \%$ mais produtivos e $8 \%$ mais baratos que aqueles gerenciados pelo Governo do Estado de Saúde ${ }^{(7)}$.

Os bons resultados alcançados pelas OSS, incitou a expansão deste novo modelo de gestão como alternativa para sanar as dificuldades encontradas por outros serviços de saúde do estado, em especial os relacionados à contratação de pessoal.

Nesta perspectiva, um município do interior paulista, no ano de 2009, em face às dificuldades encontradas no campo de recursos humanos para a atenção primária à saúde, buscou esse modelo de gestão.

A Fundação UNI (Uma Nova Iniciativa), instituída na cidade desde 1997, antes denominada como Projeto UNI na Formação de Profissionais de Saúde em União com a Comunidade, com parceira da Faculdade de Medicina de Botucatu - UNESP e da Secretária Municipal de Saúde, no apoio à formação de profissionais e desenvolvimento do sistema local de saúde e da organização da comunidade, correspondeu ao perfil solicitado, sendo qualificada como a primeira OSS de Botucatu-SP, atuando na atenção primária à saúde ${ }^{(8)}$.

Assim, este estudo fundamenta-se na concepção de que há desafios relacionados ao gerenciamento de OSS e que os discursos de membros que compõem o conselho gestor, aqui denominados como Conselho de Curadores, podem apontar caminhos necessários ao delineamento de estratégias inovadoras que auxiliem a gestão deste novo modelo.

Portanto, a realização deste se justificou, também, por suscitar, a partir da concepção dos participantes, indicativos que podem servir de apoio para melhor programação e estruturação da gestão por meio de OSS e contribuir com a investigação nesse campo.

Para tanto pergunta-se: Como tem se configurado o gerenciamento de uma OSS na perspectiva dos membros do Conselho de Curadores?

O objetivo desse estudo foi conhecer a experiência em gerenciar uma OSS segundo a perspectiva dos gestores, bem como, levantar suas potencialidades e limites.

\section{MÉTODO}

Trata-se de estudo qualitativo, do tipo "Estudo de Caso Único" tendo como objeto de estudo o 
gerenciamento da rede primária de atenção à saúde pela OSS, do município de Botucatu-SP, particularmente a Fundação UNI ${ }^{(9)}$.

Optou-se pelo Estudo de Caso, pois o mesmo apresenta-se como caminho para responder aos objetivos da pesquisa e do próprio objeto de estudo. É uma estratégia de pesquisa com dados existentes, por meio da qual o pesquisador se concentra sobre um caso, possivelmente escolhido por seu caráter típico, a fim de compreendê-lo em profundidade ${ }^{(9)}$. Caracteriza-se pela investigação profunda e exaustiva de um ou poucos objetos, possibilitando seu amplo conhecimento, tarefa dificilmente realizável por meio de outros delineamentos. Privilegia análises clínicas e longitudinais sobre mudanças e processos organizacionais, bem como, sobre política e cultura organizacional ${ }^{(9-10)}$.

Este estudo se deu no município de Botucatu, localizado na região centro-oeste do estado de São Paulo, Brasil, e possui 122.659 habitantes. Sua rede de saúde está constituída por 16 unidades e sob gestão da OSS estão 11 unidades da ESF, compondo 14 Equipes de Saúde da Família (duas unidades com duas equipes e uma equipe do Programa de Agentes Comunitários de Saúde - PACS). De forma geral, a rede de atenção primária está estruturada para prestar atendimento nas três áreas básicas, a saber: pediatria, clínica médica e gineco-obstetrícia, sendo complementada pela saúde bucal. A Fundação UNI foi qualificada como OSS no ano de 2009 para gerenciar a Estratégia Saúde da Família parte da rede de saúde do município(11-12).

A amostra por conveniência, foi composta por sete membros titulares que compõe o Conselho de Curadores, representado pela sociedade civil organizada e por gestores locais que aceitaram participar do estudo por meio do Termo de Consentimento Livre e Esclarecido, e que não foram identificados por questões éticas. Amostra por conveniência, é aquela na qual os sujeitos mais adequados são incluídos na amostra, isto é, se alinham ao objeto de estudo favorecendo o alcance dos objetivos $^{(13)}$.

Os dados foram coletados por meio de entrevista não diretiva norteada pelas questões: 1 . Como tem sido sua experiência em gerenciar uma OSS? 2. Quais são as potencialidades, desafios e limites que você encontra ao gerenciar uma OSS?

As entrevistas foram realizadas em áudio gravadas e transcritas. Os participantes da pesquisa foram identificados pelas iniciais OSS (Organização Social em Saúde), seguida do número da entrevista conforme foram realizadas (OSS1 a 7).
Para tratamento dos dados coletados utilizou-se a análise de conteúdo na abordagem representacional do tipo temática proposta por Bardin ${ }^{(14)}$, que tem como objetivo atingir os significados manifestos e latentes no material pesquisado, os discursos gerados a partir das entrevistas ${ }^{(14)}$.

No primeiro momento, realizou-se a transcrição dos discursos na íntegra, seguindo-se da leitura flutuante e exaustiva dos dados. Esta etapa, demominada préanálise, exige que o pesquisador tenha a pergunta do estudo e seus objetivos claros, pois, são necessários para direcionar a análise. O contato com os discursos permite ao pesquisador organizar as ideias dos entrevistados, seus significados, além de formular hipóteses. Nesta fase não são permitidas exclusões de nenhum seguimento dos discursos ${ }^{(14)}$.

A segunda fase, chamada de exploração do material, permitiu conhecer os discursos em profundidade. Para delimitação do contexto, realizou-se recortes do texto, os quais permitiram a elaboração de categorias temáticas, que surgiram a partir do corpus de análise ${ }^{(14)}$.

O tratamento dos resultados obtidos caracteriza a teceira e última etapa do processo onde o pesquisador deve realizar as inferências e interpretações. Procedeuse a condensação das categorias que surgiram, momento importante que requer intuição, reflexão e crítica carregados de significado, pois possibilita ir além do que foi dito ${ }^{(14)}$.

Ressalta-se que, ao trabalhar com a análise de conteúdo, todo zelo deve ser tomado com a descrição e execução de cada uma das fases da análise e, por mais que se preservem a flexibilidade, intuição e a criatividade, evidencia-se como forma de gerar confiabilidade e validade ${ }^{(14)}$.

A pesquisa foi aprovada pelo Comitê de Ética em Pesquisa da Universidade Estadual Paulista- UNESP (Of. 4115-2012), conduzida após o Consentimento Livre e Esclarecido dos participantes.

\section{RESULTADOS E DISCUSSÃO}

A partir da análise de conteúdo e da organização dos dados emergiram quatro categorias temáticas que revelaram a experiência dos membros do Conselho de Curadores em participar do gerenciamento da OSS na sua primeira gestão: o processo histórico, as transformações sociais e a OSS local; o contrato de gestão da OSS: agilidade e novos aprendizados; Organizações Sociais de Saúde: uma nova possibilidade de gestão e limitações no gerenciamento e nos mecanismos de controle da OSS local. 


\section{O processo histórico, as transformações sociais e a oss local}

A reflexão sobre as relações entre o público e o privado remonta ao Direito Romano e ganha novos contornos com o desenvolvimento do Estado moderno, estimulando o debate sobre os novos modelos para a administração pública em decorrência de novos contextos e novas exigências ${ }^{(2,4)}$.

A partir da Constituição Federal de 1988, conceitos de descentralização e municipalização da gestão da atenção à saúde fortaleceram-se. Porém, dentre as atribuições legais previamente definidas, não se estabeleceu nitidamente como deveriam ser as relações entre os entes federados no âmbito da repartição dos recursos, procedendo assim na década de 90 uma crise fiscal do setor ${ }^{(15)}$.

Frente as dificuldades encontradas pelo Estado, sendo de relevância a escassez de recursos, surgem novos modelos de gestão com o objetivo de atender as necessidades encontradas ${ }^{(4,16)}$.

Por esta razão, importa conhecer o processo histórico e as transformações sociais ocorridas no município estudado, ao mesmo tempo que o debate estadual ganha força. Esse processo foi apoiado pela rede local de saúde, que à época era constituída pela Universidade Estadual Paulista - UNESP, Secretaria Municipal de Saúde e entidades filantrópicas.

Entende-se por transformação social as alterações que ocorrem em uma determinada sociedade, instituições sociais ou ainda nas relações entre as instituições e que afetam o desenvolvimento local ${ }^{(17)}$.

O serviço de saúde do município de Botucatu-SP, no início do ano 2000, encontrou-se em situação semelhante a de outros municípios brasileiros, limitados pela Lei de Responsabilidade Fiscal, que prejudicava a qualidade do serviço prestado, como evidenciado pelos gestores:

[...]pela lei de responsabilidade fiscal o nosso quadro de pessoal estava praticamente próximo do seu limite não tendo condições de contratar mais pessoas para o desenvolvimento do sistema de saúde. Assim, o município procurou alternativas para resolver o problema [...] (OSS1)

$\mathrm{Na}$ busca por novas alternativas de gestão, o município instituiu convênio com instituição filantrópica para conter as limitações de recursos sofridas no financiamento de serviços essenciais à população. Entretanto, ao longo dos anos, essas relações não foram regulamentadas de maneira efetiva como destaca o fragmento:

[...] essa instituição não tinha autonomia e independência, era um mascaramento, vamos dizer assim, de uma relação de terceirização totalmente sem controle. (OSS2)

Dessa forma, a implantação do Projeto UNI - uma iniciativa na formação de profissionais de saúde em união com a comunidade - apoiado pela Fundação W.K.Kellogg, se deu em Botucatu no ano de 1992, como iniciativa inédita local.

Em 1997, como estratégia para o fortalecimento do cárater tripartite da iniciativa UNI, assim como para construção de parcerias permanentes, tal projeto se consolidou como Fundação UNI, apoiada no tripé: universidade, sistema local de saúde e sociedade civil organizada. Sua missão era o apoio permanente à formação de profissionais, do desenvolvimento do sistema local de saúde e da organização da comunidade ${ }^{(8)}$.

A contento, entre 2002 e 2009, o município manteve parceria com instituição filantrópica local e, por problemas legais regulamentares, foi interrompido.

Com o objetivo de atender as necessidades encontradas, a Fundação UNI, pode ser qualificada como OSS e, no ano de 2009, por meio de processo licitatório, começou a gerenciar a atenção primária à saúde local ${ }^{(8)}$.

[...] resolvemos transformar uma relação entre o poder público (secretaria de saúde) e instituições privadas (Fundação UNI) que já existiam no município, fazendo com que essas relações se dessem através de um contrato de gestão como as OSS [...] (OSS2)

Diversas exigências são solicitadas para qualificação das OSS, entre elas as que permitiram a qualificação da Fundação UNI, tais como: experiência comprovada de mais de 05 anos na administração de serviços de saúde, disponibilização dos dados gerenciais ao Tribunal de Contas do Estado, atendimento exclusivo a pacientes do Sistema Único de Saúde (SUS) e a constituição de um contrato de gestão entre o poder público e a OSS, com o objetivo de regulamentar a relação entre ambos ${ }^{(16)}$.

\section{O contrato de gestão da OSS: agilidade e novos aprendizados}

Evidencia-se a importância em gerenciar o contrato de gestão de uma OSS, sendo destacado fortemente 
pelos participantes da pesquisa. A literatura aponta que "o contrato de gestão configura-se como dispositivo de acompanhamento e avaliação do desempenho institucional da entidade contratada e de aperfeiçoamento contínuo das suas relações de cooperação, supervisão e fiscalização com o poder público"(4).

Trata-se de um compromisso institucional entre o Estado e as OSS, devendo respeitar os princípios da legalidade, da impessoalidade, da moralidade, da publicidade e da economicidade. Este deve permitir a avaliação e controle de metas de desempenho, com prazos, limites e critérios previamente acordados ${ }^{(2,16)}$.

Para o poder público, o contrato de gestão é um instrumento de implementação, supervisão e avaliação das políticas públicas, enquanto que, para as organizações, trata-se de um instrumento de gestão estratégica, pois oferece informações como recursos disponíveis e objetivos pactuadas ${ }^{(3)}$.

A respeito do contrato de gestão, os participantes relatam:

O contrato de gestão significa transferir a execução de atividades com parceiros, mas, depois regular a produção desse serviço, controlar a produção para que aquilo que é feito corresponda com aquilo que a administração pública gostaria que fosse feito, dentro do plano municipal de saúde, do próprio mecanismo regulador da saúde [...] (OSS2)

[...] na condição de membro do Conselho de Curadores nós temos um norte. A Fundação

UNI não faz o que bem entende, ela é regida por um estatuto, e ao mesmo tempo tem que executar aquilo que está previsto no contrato de gestão. (OSS3)

Dessa forma, o contrato de gestão encontra respaldo nos discursos, pois, determina metas de produção e qualidade para um período acordado com o correspondente valor a ser repassado, além de outras atribuições e dos mecanismos de acompanhamento incluindo possibilidades de ajustes por meio de aditivos contratuais $^{(2,4)}$.

A parceria entre o poder público e a OSS municipal facilitou os processos de trabalho e gestão, evidenciados pelos fragmentos abaixo:

[...] a administração da nossa rede ganhou uma agilidade tremenda com esse contrato de gestão e o mais importante com racionalização do uso dos recursos, porque o tribunal de contas cobra muito isso e nós temos conseguido demonstrar que é mais vantajoso pra administração pública manter esse contrato de gestão. (OSS2)

A maior contribuição da OSS é essa agilidade que ela tem de tomar decisão, de direcionar. (OSS4)

Os objetivos e metas da OSS são determinados no contrato de gestão, com a intencionalidade de propiciar a participação mais efetiva e avaliação dos resultados com mais propriedade. Assim sendo, há maior autonomia dessas organizações, dando mais agilidade ao setor, como podemos constatar:

[...] nós conseguimos fazer concursos, conseguimos contratar com bastante agilidade, comprar com bastante agilidade materiais de enfermagem e materiais hospitalares [...] (OSS2)

[...] você consegue ser ágil, consegue ter agilidade nas decisões, você não depende muito das leis que amarram um pouco a parte do estado, você consegue tomar decisões rápidas, você consegue, assim, mudar o curso das coisas $[\ldots]$ (OSS4)

O contrato de gestão é gerenciado por um Conselho de Curadores, órgão máximo da OSS, com funções deliberativas, normativas e de fiscalização da administração. É composto por membros de órgão públicos envolvidos e representantes da sociedade ${ }^{(18-19)}$.

Este contrato é uma oportunidade de negociação permanente de metas e de redirecionamento de políticas e objetivos pelos gestores envolvidos.

Os membros do conselho se mostram satisfeitos com esta nova modalidade de gestão:

[...] Você vive uma série de experiências que são gratificantes, quando analisa os indicadores de produção, os indicadores de saúde do município, você percebe que está havendo uma transformação, com a queda da morte neonatal, a incidência de certa doença que diminuiu [...] (OSS1)

A participação da sociedade se faz indispensável, já que é a sociedade civil organizada que viabilizará o controle social sobre o Estado. Essa participação referese à relação entre os órgãos governamentais e movimentos sociais. No Brasil esta relação está aprovada desde 1990 - Constituição Cidadã ${ }^{(20)}$.

Em relação a esta participação os representantes da OSS afirmam que a nova experiência é válida, porém, apresenta dificuldades: 
[...] apesar de ter representantes de funcionários muitas vezes não temos muita voz sobre alguns assuntos, infelizmente somos contados só como mais um voto pra tomar algumas decisões, então falta adequar um pouquinho isso [...] (OSS5)

[...] A OSS tenta ter membros da comunidade cada vez mais participativos, ela traz o interesse da comunidade dentro dela $[\ldots]$ (OSS6)

Historicamente a participação social é restrita e pouco efetiva, fato que pode estar atribuído a centralização do poder e a visão de que as ações são apenas de fiscalização, além de distanciar a população por seu caráter burocrático e hierárquico evidentes ${ }^{(19)}$.

Entretanto, as ações de fiscalização executadas pela Secretaria Municipal de Saúde e pelo Tribunal de Contas do Estado, mantém a proposta de aumentar a eficiência e a qualidade dos serviços de saúde, atendendo melhor o cidadão, com menor custo, garantindo seu controle em caráter complementar ${ }^{(2,8)}$.

Assim, a participação social deve ser fomentada sistematicamente, melhorando os processos democráticos e de cidadania, incentivando a participação de todos e valorizando as ideias, sugestões e desejos, garantindo assentos permanentes nessas organizações $^{(19)}$.

\section{Organizações Sociais de Saúde: uma nova possibilidade de gestão}

Alguns estudos ${ }^{(21-22)}$ apontam que a gestão por meio de OSS proporciona avanços nos serviços de saúde e, nesse sentido, a gestão de recursos humanos ganha destaque, já que esta não fica sujeita as normas do Regime Jurídico Único dos Servidores Públicos, que deve ser realizado em geral por meio de concursos públicos caracterizados por processos lentos e altamente burocráticos ${ }^{(21-22)}$.

Nesta perspectiva, a gestão por meio de OSS, tem como potencial regulamentar seu próprio quadro de pessoal, definir as formas de ingresso e permanência no serviço, propiciar a agilidade e qualidade das contratações, manutenção e desligamento de funcionários, que estão sujeitos ao regulamento próprio, caracterizados pelo regime da CLT.

Além disso, possuem autonomia nos processos administrativos, orçamentários, financeiros e de compras, como relatado pelos gestores:

[...] eu consigo contratar pessoas de uma maneira mais rápida, consigo fazer uma política de compra mais adequada, consigo discutir e ser um facilitador [...] (OSS1)

[...] nós temos uma gestão de recursos humanos, os processos de compra, os processos licitatórios são guiados pela impessoalidade, a economicidade, a racionalidade, então, nós conseguimos responder as necessidades, por exemplo, de contratação de pessoal e flexibilização dos vencimentos. (OSS7)

Dessa forma, há melhorias consideráveis em relação à autonomia administrativa e financeira das OSS no que tange a contratação de recursos humanos e compras/abastecimento de materiais e medicamentos, otimizando tempo e ajustando seu déficit nos arranjos organizacionais em saúde ${ }^{(22)}$.

O grau de autonomia concedido e os tipos de controle adotados formam uma tênue linha divisória, podendo destacar um ou outro aspecto, de acordo com o modelo do contratado ${ }^{(23)}$. A busca puramente por resultados gera risco de ineficiência dos recursos públicos e, na outra ponta, controles estritos podem levar ao excesso de formalidades processuais, ingerência do poder público à OSS, dificultando seu processo de trabalho.

A Secretaria do Estado de São Paulo, no ano em que iniciou o gerenciamento dos serviços de saúde a partir das OSS, realizou pesquisas de opinião onde o índice de satisfação do usuário ficou entre $80 \%$ e $90 \%{ }^{(24)}$. Nesse sentido, do ponto de vista dos gestores da OSS, depreende-se que as melhorias ocorridas na saúde municipal após a mudança no modelo de gestão, também satisfazem os usuários.

[...] nossa cidade tem um gerenciamento muito mais efetivo, nós temos menos queixas do que temos em outras cidades e eu imputo esse menor número de queixas ao melhor gerenciamento que é feito pela OSS [...] (OSS6)

[...] o município foi indicado pelo MS como a sexta cidade no país em termos de organização de sistema de saúde. No estado de $S P$, eu acho que é a quarta se não me falha a memória, quer dizer, esses bons resultados não existiriam, com certeza, se nós não tivéssemos viabilizado a estrutura de uma oS junto com a prefeitura de Botucatu [...] (OSS1)

Em três anos, a OSS estudada tem conseguido atingir as metas propostas, evidência primordial para avaliação da qualidade e eficiência do contrato de gestão vigente, destacado nesse fragmento: 
Os principais resultados que conseguimos em dois anos foram: do PSF, nós tínhamos oito equipes, hoje nós temos 14. Em número de funcionários, para todos os setores da área de saúde, desde vigilância epidemiológica até assistência direta ao paciente, feita por médicos, enfermeiros, fisioterapeutas, psicólogos e outros, saímos de um grupo de 215 para 440 funcionários, isso é outro salto de qualidade, o número de atendimentos também seguiu um aumento proporcional. (OSS1)

Apesar das críticas quanto ao modelo de gestão das OSS, vê-se melhora significativa na qualidade do serviço prestado no município, lembrando que este estudo parte da avaliação dos membros do Conselho de Curadores da Fundação UNI, sustentados por indicadores de produção e qualidade locais.

\section{Limitações no gerenciamento e nos mecanismos de controle da oss local}

Todo serviço oferecido à sociedade deve ter alternativas para realizar o controle dos serviços prestados, sendo sua principal dificuldade a determinação dos mecanismos de controle que são exercidos principalmente pelo Estado, mercado e/ou sociedade civil(21).

No modelo das OSS, estes mecanismos devem estar presentes desde a sua qualificação até a prestação de contas. Em geral possuem duas vias de controle: o Conselho Administrativo, chamado neste caso de Conselho de Curadores, e o Contrato de Gestão, já citado anteriormente, que determina o controle focado nos resultados, devendo prestar contas da parte financeira, recursos humanos e patrimônio, além dos resultados alcançados, comparando-os com as metas pactuadas no contrato de gestão.

Corroborando com dados da literatura, este estudo evidenciou que os mecanismos de controle por meio dos indicadores de saúde provenientes de dados do sistema de informação, são ferramentas altamente eficientes no quesito de transparência das OSS, porém, devem ser por estas aprimoradas ${ }^{(3,21)}$.

Existe um controle exercido pela própria OSS, interno e autônomo, visando melhorias contínuas da aplicação dos recursos de acordo com a legislação que regula a parceria com o poder público ${ }^{(21)}$.

As limitações são muitas, nós temos que aperfeiçoar, aprimorar mais os mecanismos de controle. Nós herdamos uma estrutura, por exemplo, de registro de informações muito limitado, um sistema de informações muito limitado, então, hoje se eu quiser aprimorar os controles, sobre o que é produzido nas unidades, eu ainda tenho dificuldade. (OSS2)

Nota-se que, o sistema de controle informatizado ainda encontra limitações para demonstrar transparência e alicerçar plenamente a tomada de decisões do Conselho de Curadores. Porém, este deve proporcionar aos seus usuários uma interface amigável que fomente sua alimentação diária ou mensal, fato que a OSS tem se preocupado em resolver junto às unidades de saúde gerenciadas $^{(21)}$.

Entretanto, o controle da gestão das OSS deve ser realizado por parte da administração, em caráter optativo, por meio de avaliações diretas com objetivo de elaborar melhorias e identificar riscos relacionados ao contrato de gestão.

E ainda, obrigatoriamente, avaliações externas devem ser feitas de forma que os serviços prestados por essas organizações sejam fiscalizados pelo Poder Público, por meio do Ministério da Saúde e do Tribunal de Contas $^{(3)}$.

Em termos legais, os gestores de uma OSS são responsabilizados em sua pessoa física pelo contrato firmado, conforme o interesse público, em qualquer momento e após seu encerramento pelo prazo de até cinco anos ${ }^{(25)}$.

A responsabilidade é grande, você é auditado pelo tribunal de contas, passa a responder fisicamente, pessoa física, pelos atos da fundação. (OSS7)

Desta maneira, o sucesso desse controle depende do Estado manter-se ativo em seu papel de fiscalizador, uma vez que o descumprimento das ações previamente acordadas, podem resultar em sanções punitivas e corretivas.

O uso de ferramentas que garantam a transparência, em geral, são escassos em muitas OSS, a não obrigação da publicação de dados e resultados em locais de fácil acesso, impossibilita a real participação social.

Neste contexto, a Lei de Responsabilidade Fiscal (Lei n 101/2000), a Lei da Transparência (Lei n¹31/2009) e a Lei de Acesso à Informação (Lei no 12.527/2011), podem ser caracterizadas como estratégias para viabilizar o contexto de transparência das OSS, necessários para atender a importância da sociedade, conhecer seus processos e atividades ${ }^{(21)}$. 
Outra importante limitação é a escassez de recursos financeiros, citada pelos participantes. Apesar de sua autonomia administrativa e financeira, esta deve respeitar os limites orçamentários estabelecidos pelo Estado. Porém, possuem a liberdade de fazer os arranjos institucionais necessários para o melhor uso do recurso disponível ${ }^{(4)}$

Para tal, o contrato de gestão deve prever recursos necessários para 0 desenvolvimento das metas propostas.

O maior desafio são os recursos, nunca são suficientes, no caso de OS depende de recurso do estado, e o recurso do estado a gente sabe como é, ele é um bolo pra dividir pra muitos [...] (OSS4)

Trabalhar dentro da ótica da OS é trabalhar com menos recursos, o desafio é sempre como fazer pra executar as tarefas com menos gasto. (OSS6)

A estruturação dos processos de trabalho nesse modelo de gestão devem apoiar-se no equilíbrio entre as metas e os recursos necessários. É recomendável, que se tenha uma previsão de alteração das metas e custo do contrato de gestão, como forma de buscar a eficiência do serviço prestado, periodicamente ${ }^{(4,25)}$. Nesse contexto, a OSS municipal mantém um diálogo permanente com a secretaria municipal de saúde, para que as metas pactuadas sejam atendidas a contento, sem prejuízo à população.

A complexidade do Sistema Único de Saúde (SUS) e a diversidade de modelos de gestão, indicam a necessidade de novas alternativas para a solução dos problemas dos serviços de saúde. O modelo de gestão das OSS tem sido capaz de gerar aprendizados organizacionais num contexto de mudanças onde se espera aprimoramento dos mecanismos de controle, tomada de decisão descentralizada, participativa e resolutiva, que atenda às necessidades de preservação do patrimônio público(22).

\section{CONCLUSÃO}

O uso do Estudo de Caso, permitiu o alcance do objetivo do estudo, de forma que foi possível conhecer a

\section{REFERÊNCIAS}

1. Weichert MA. Fundação estatal no serviço público de saúde: inconsistências e inconstitucionalidades. Rev de Direito Sanitário. 2009;10(1):81-97.

2. Coelho RC. O público e o privado na gestão pública. $2^{\mathrm{a} e d}$ Florianópolis: Departamento de Ciências da Administração/UFSC; 2012. experiência dos gestores no gerenciamento da OSS, no contexto de uma recém-implantação no município estudado, assim como suas potencialidades e limites.

O estudo apontou como potencialidades, a agilidade dos serviços com destaque à capacidade de resolver problemas de recursos humanos, compras de insumos e medicamentos com a agilidade esperada e custo reduzido, atendendo aos objetivos da OSS, demonstrando uma melhor organização dos processos de trabalho com a utilização de gestão moderna e resolutiva.

Destaca-se ainda a importância do contrato de gestão, o qual deve conter metas claras e concisas, respeitando as avaliações sistematizadas que se fazem necessárias para o bom funcionamento da oSS e o controle social necessário.

Foi de extrema relevância clarificar as limitações enfrentadas pelos gestores no gerenciamento do contrato de gestão da OSS, pois, sendo um modelo de gestão novo, apresenta-se como um desafio frente a necessidade em aprimorar mecanismos de controle que garantam transparência.

A necessidade de sistemas de informação especializado se faz indiscutível, de forma que facilite o uso de indicadores para tomada de decisões e que possibilite o controle pelos órgãos competentes, sendo a chave para o controle social. Além disso, importa salientar que os recursos financeiros sejam periodicamente revistos e corrigidos para atender a necessidade da rede de saúde local.

As análises e reflexões sobre osS devem ser consideradas como oportunidades de melhoria do sistema de gestão em saúde, experiências municipais com modelo de gestão de OSS, principalmente pela característica de gerenciar a atenção primária à saúde, podem ser replicadas em outros cenários, observados os objetivos e metas do contrato de gestão garantindo eficiência e transparência pública.

Espera-se que essa pesquisa possa subsidiar novos estudos e contribuir para novas aprendizagens acerca dos modelos de gestão em saúde, com o propósito de oferecer melhorias na prestação de serviços por meio de OSS à sociedade. 
SUS. $2^{a}$ ed. Florianópolis: Departamento de Ciências da Administração/UFSC; [Brasília]: CAPES:UAB; 2012.

5. Lei Complementar n. 846, de 4 de junho de 1998. Dispõe sobre a qualificação de entidades como organizações sociais. Diário Oficial do Estado de São Paulo. Jun 5. 1998.

6. Taborda W, Gomes MC, Paschoa NF, Barata LRB. Organizações Sociais de Saúde do Estado de São Paulo: Funcionamento e Resultados. In: Terceiro Setor e Parcerias na área da Saúde. Ed. Fórum. Belo Horizonte. 2011.

7. World Bank, Brazil: Enhancing Performance in Brazil's Health Sector: Lesson form innovations in the State of São Paulo and the City of Curitiba. March 29, 2006, Brazil Country Management Unit Poverty Reduction and Economic Management Unit Latin America and the Caribbean Region.

8. Prefeitura Municipal de Botucatu. Contrato n. 423/09. Contrato de Gestão. Contrato que entre si celebram o município do Botucatu e a Fundação UNI, qualificada como Organização Social, com vistas à regulamentação do desenvolvimento de ações e serviços de saúde no âmbito do município de Botucatu-SP. Botucatu; 2009.

9. Yin RK. Estudo de caso: planejamento e métodos. 4a. ed. Porto Alegre: Bookman; 2005.

10. Anselmo E. Mudanças estratégicas nas empresas: um estudo de caso. [dissertação]. São Paulo: Faculdade de Economia, Administração e Contabilidade da Universidade de São Paulo; 1995.

11. Secretaria Municipal de Saúde de Botucatu. Plano Municipal de Saúde: Gestão 2013-2016. Botucatu; 2013.

12. Spagnuolo RS. Coordenar equipe multiprofissional: um desafio para o enfermeiro do Programa Saúde da

Família. [dissertação]. Botucatu: Departamento de Saúde Pública, Faculdade de Medicina de Botucatu: Universidade Estadual Paulista "Júlio de Mesquita Filho"; 2006.

13. Fontanella BJB, Ricas J, Turato ER. Amostragem por saturação em pesquisas qualitativas em saúde: contribuições teóricas. Cad Saude Publica. 2008; 24(1):17-27.

14. Bardin L. Análise de conteúdo. Lisboa: Edições 70; 2009.

15. Costa LS, Marques P, Borges TR. A Prestação pelo setor público não estatal dos serviços de saúde pública: análise de hipóteses e potencialidades. Rev do Servidor Público.

2010;61(3):249-297.

16. Tibério AA, Souza EM, Sarti FM. Considerações sobre Avaliação de Estabelecimentos de Saúde sob Gestão de OSS: o Caso do Hospital Geral de Grajaú. Saúde Soc. 2010;19(3):557568.

17. Castles S. Estudar as transformações sociais. Sociol Probl Prát. 2002;40:123-48.

18. Fundação UNI. Estatuto da Fundação UNI. Botucatu; 1997. 19. Fundação UNI [Internet]. História da Fundação UNI [acesso em 20 Maio 2012]. Disponível em:

http://www.fundacaouni.org.br/quem_somos/historia

20. Shimizu HE, Pereira MF, Cardoso AJC, Bermudez XPCD. Representações Sociais dos Conselheiros Municipais acerca do Controle Social em Saúde no SUS. Ciências \& Saúde Coletiva. 2013;18(8):2275-2284.

21. Camargo TA, Prado O, Bernardo R, Lara ACHS. Controle e Transparência no Modelo de Organizações Sociais de Saúde do Estado de São Paulo. In: Painel 13/050. VI Congresso CONSAD de Gestão Pública, 2013, Brasília, Brasil[Internet]. 2013 [acesso em: 24 mar 2014]. Disponível em: http://consadnacional.org.br. 22. Barbosa NB, Elias PEM. As organizações sociais como forma de gestão público/privado. Ciência \& Saúde Coletiva.

2010;15(5):2483-2495.

23. Bernardo R, Martins HF. As OSCIPs em Minas Gerais: tensões entre autonomia e controle. In: XVII Congresso Internacional del CLAD sobre la Reforma del Estado y de la Administración Pública, 2012, Cartagena, Colombia [Internet]. 2012 [acesso em: 24 mar 2014]. Disponível em:

http://www.dgsc.go.cr/dgsc/documentos/cladxvii/bernaren.pdf 24. Araújo MCD. Contrato de gestão hospitalar implantado no Estado de São Paulo, sob a égide da Lei Complementar n.846, de 04.06.98 [dissertação]. Rio de Janeiro: Fundação Getúlio Vargas; 2008.

25. Tribunal de Contas do Estado de São Paulo. Manual básico: repasses públicos ao terceiro setor. São Paulo; 2007.

Artigo recebido em 31/01/2013.

Aprovado para publicação em 23/04/2014.

Artigo publicado em 30/09/2014. 